\title{
IDEOLOGÍA Y ANTI-GLOBALIZACIÓN: UNA APROXIMACIÓN AL DISCURSO DE LA VÍA CAM PESIN A
}

\author{
JoRGE LAZO CIVIDANES \\ Universidad de Salamanca, España
}

\begin{abstract}
Resumen
Siguiendo técnicas de análisis de contenido, este trabajo aborda el discurso político de la vía campesina, una de las organizaciones más grandes opuestas a la llamada globalización. Nuestro objetivo es saber si los elementos estructurantes de la ideología se encuentran presentes en el discurso político de esta organización, en qué términos se manifestarían y de acuerdo a qué lógica. Pretendemos, asimismo, contribuir a llenar un vacío en el estudio y comprensión de los discursos que movimientos como la vía campesina están produciendo, y someter al análisis empírico una teoría de la ideología vinculada a las luchas por el poder simbólico.
\end{abstract}

\section{Abstract}

According to discourse analysis methods, we are able to use this approach to the political discourse of "La Via Campesina", one of the largest partisan groups against globalisation. Our aim is to know which structural elements of the ideology are present in the organisation's discourse, in which terms they are delivered if present, and what logic they follow. We also aim at filling a vacuum in the study of discourse analysis, understanding this kind of discourse, and empirically testing an ideology theory linked to the symbolic power struggles.

PALABRAS CLAVE • Movimientos Sociales • Ideología • Globalización • Discurso Político - Representaciones • Poder Simbólico

\section{INTRODUCCIÓN}

Sabemos que el surgimiento de los movimientos sociales tuvo su origen en los conflictos y oportunidades derivadas del proceso de consolidación de los Estados-nación, como un modo de oposición ciudadana (Tarrow, 1999: 82). Según los historiadores, estos movimientos presentan una continuidad en el tiempo, que no excluye los matices propios de las distintas épocas y circunstancias, ni niega influencias diversas sobre los modos de realizar la protesta (Ibarra y Tejerina, 1998: 9). Las revoluciones burguesas, los procesos de democratización, el desarrollo del Estado de bienestar y el aumento de la prosperidad económica, han sido consideradas causas de la movilización. Hoy tenemos en la llamada globalización la primera referencia para el estudio del tema por los cambios y las vastas reacciones que ha producido ${ }^{1}$.

Como Tarrow sostiene, "hubo un tiempo en el que el Estado nacional podía ser el marco adecuado para interpretar 0 predecir las actividades desplegadas por los movimientos sociales, pero esta posibilidad se reduce día a día. Esto es así porque la movilización se da en múltiples niveles y en sus movimientos existen diversas facciones, que experimentan grandes variaciones dependiendo de los ciclos de protesta y que, además, tienen vínculos transnacionales cada vez más amplios" (Tarrow, 1999: 88). 
Para las ciencias sociales, el estudio de los nuevos movimientos sociales ${ }^{2}$ ofrece a los investigadores "un lugar donde espiar lo que está pasando en nuestra sociedad moderna" (Mardones, 1996: 42). En este sentido, a nuestro juicio, campo privilegiado para tales observaciones son los llamados movimientos anti-globalización, dada su vitalidad, las dimensiones que han alcanzado y el carácter de sus protestas. En particular, consideramos que el discurso político de estos movimientos resulta un ámbito de estudio de enorme interés e importancia en el conocimiento y comprensión de las luchas por legitimar distintas representaciones de la realidad que involucran relaciones de poder en el mundo contemporáneo.

Se trata de un campo curiosamente inatentido por los estudios politológicos, en parte quizás por prejuicios o lecturas que atienden a los aspectos más evidentes, sin que para ello medie, por cierto, ninguna aproximación sistemática. Aunque se reconoce a los llamados grupos antiglobalización su capacidad de movilización y presencia internacional, no ha sido suficiente para motivar estudios adecuados sobre los discursos que estas organizaciones están produciendo. Este trabajo tiene su origen en esta carencia.

Consideramos que el debate que estos movimientos están impulsando es la arena donde se libra una amplia lucha ideológica, donde tiene lugar un caudaloso flujo y reflujo de viejas y nuevas ideas políticas, debate frente al cual los partidos políticos (a igual que otras instituciones) lucen más bien rezagados y desbordados en iniciativa, intentando con poco éxito articular respuestas que capitalicen estas demandas y desafecciones. Por ello nos hemos interesado en el tema, particularmente en el discurso político de la vía campesina, una de las organizaciones de mayor presencia y actividad entre lo grupos opositores a la llamada globalización ${ }^{3}$.

Como frecuentemente ocurre con estos movimientos, la vía campesina se interesa en la construcción de alianzas con otras fuerzas sociales, económicas y políticas en todo el mundo, haciendo de

2 Desde la década del 70 se habla de viejos y nuevos movimientos, pero en realidad hay muchos y diversos (Mees, 1998: 293). Aquí entendemos que un movimiento social es "una colectividad de personas unidas por una creencia común (ideología) y por la determinación de desafiar el orden existente en pos de objetivos implícitos en esa creencia fuera de los cauces institucionalizados de intermediación de intereses" (Mardones, 1996: 14). Se trata de "un actor colectivo que interviene en el proceso de cambio"; un actor que tiene una estructura (grupos con base en una integración simbólica) y unas metas (Raschke, 1994: 123). Además, los movimientos sociales serían, por una parte, el producto de "la incapacidad del sistema institucional establecido para encontrar respuestas a los problemas articulados en los movimientos sociales" (Raschken, 1994: 126); y, por la otra, una forma específica de institución social, en la medida que están constituidos por un conjunto de normas preestablecidas, que formal o informalmente constituyen una guía para la acción (lbarra y Tejerina, 1998: 12).

3 Toda la información referente a esta organización, los documentos y las citas, han sido tomadas de la página web de la vía campesina (www.viacampesina.org). La Vía Campesina es un movimiento social presente en cuatro continentes. Reúne a campesinos sin tierra, pequeños y medianos productores, trabajadores agrícolas, mujeres rurales y pueblos indígenas que, como tantos otros, luchan "contra la Globalización de la economía y el hambre en el mundo y consecuentemente contra el modelo Neo-Liberal", por una sociedad que "recoja las aspiraciones y esperanzas de los más débiles". En número superior a 60, organizaciones de caracter nacional o regional se integran en el movimiento, distribuidas en ocho regiones (Europa Este, Europa Oeste, Noreste y Sudeste de Asia, Asia del Sur, América del Norte, El Caribe, Centroamérica y América del Sur). Su origen data de 1992, momento en el que diversos líderes campesinos centroamericanos, norteamericanos y europeos se reunieron en Managua (Nicaragua), en el marco del Congreso de la Unión Nacional de Agricultores y Ganaderos. Un año después, en mayo de 1993, tendría lugar la Primera Conferencia de La Vía Campesina, en Bélgica, donde se constituye como organización de carácter mundial y se definen los primeros lineamientos estratégicos de su trabajo, así como sus estructuras. Desde entonces, La Vía Campesina ha experimentado un proceso de expansión que la ha convertido en una organización compleja, multicultural, con una amplia cobertura geográfica; además, reclama para sí "la más alta representatividad de las y los pequeños y medianos productores a nivel mundial". 
la lucha contra el neoliberalismo y la búsqueda de propuestas alternativas el gran punto de encuentro de la contestación y la protesta. Los temas de trabajo para todas sus organizaciones afiliadas, los siete ejes claves de su discurso, son: Soberanía Alimentaria y Comercio Internacional, Lucha por la Reforma Agraria y los Cambios Sociales en el Campo, Género, Derechos Humanos, Agricultura Campesina Sostenible, Biodiversidad, Bioseguridad y Recursos Genéticos y Derechos de Agricultores y Comunidades Rurales.

En este estudio, concretamente, nos interesa saber si los elementos estructurantes de la ideología se encuentran presentes en el discurso político de la vía campesina. Y si lo están, en qué términos se manifiestan y de acuerdo a qué lógica. En consecuencia, el objetivo de este trabajo es poder brindar respuestas a estas cuestiones, partiendo de la premisa de que la ideología estructura y articula los discursos políticos a partir de unos determinados elementos presentes en él.

Constituye éste un estudio de caso y, en tal sentido, tiene sobre todo un carácter exploratorio y descriptivo, propio de un acercamiento inicial a un fenómeno complejo y poco estudiado. La técnica para la recogida de los datos y su tratamiento es el análisis de contenido, dentro del paradigma cualitativo. Pensamos que lo ideológico subyace, de modo que cuando nos aproximamos a ello debemos hacerlo consciente de que, en efecto, frecuentemente, "lo visible, eso que está inmediatemente dado, oculta lo invisible que lo determina" (Bourdieu, 1987: 151).

El trabajo comienza con el establecimiento de las relaciones básicas de la ideología con el lenguaje y el discurso político. Seguimos con una descripción sumaria de algunos aspectos generales de la ciencia que conforman el marco epistemológico en el que se ubica este estudio y un breve repaso de las premisas metodológicas que fundamentan la investigación cualitativa. Luego damos cuenta de las técnicas de análisis de contenido a las que se sometió el discurso político de "La Vía Campesina" y los resultados de ello. Al final, ofrecemos algunas conclusiones generales ${ }^{4}$.

\section{IDEOLOGÍA, LENGUAJ E Y DISCURSO}

La ideología ha sido -y es- un ámbito de estudio polémico y complicado. Su larga historia, llena de acepciones y aproximaciones diversas, de cuestionamientos, óbitos y renancimientos, dan cuenta de ello. En muchos de estos episodios, la ideología (el ámbito de estudio) ha terminado siendo víctima de sí misma (el fenómeno). Negándolo o utilizándolo interesadamente, algunos sociólogos y politólogos han contribuido a oscurecer el término, a hacerlo confuso y, en consecuencia, su estudio ha padecido el desaliento y la indiferencia de una parte importante del mundo politológico. No obstante, con el progresivo desarrollo de teorías que rescatan la significación e importancia de las relaciones simbólicas de poder, del mundo de las representaciones sociales y del discurso, el concepto de ideología se ha revitalizado.

Nuestras premisas en torno a la ideología y su relación con el lenguaje y el discurso político son las siguientes. Entendemos que la vida social es simbólica y, por tanto, "susceptible de distorsión" (Ricoeur, 1997: 28). Es esto lo que hace posible el hecho ideológico como forma de conocimiento de la realidad. Básicamente, la ideología constituye "sistemas de creencias explícitas, integradas y coherentes, que justifican el ejercicio del poder, explican y juzgan los acontecimientos históri-

Todas las citas hechas en esta investigación provenientes de textos en francés han sido traducidas por el autor para facilitar la lectura del trabajo. 
cos, identifican lo que está bien y lo que está mal en política, definen las relaciones entre la política y otros campos de actividad y suministran una guía para la acción" (Bobbio, 1998: 758).

Adicionalmente, la ideología comporta creencias sociales compartidas por un grupo, creencias que controlan y organizan el conocimiento y las opiniones (actitudes) específicas de éste (Van Dijk, 1999: 72). Por lo tanto, debemos comprender la ideología en los cuatro planos que la componen: el simbólico (representaciones) ${ }^{5}$, el colectivo (grupo), el conductual (acción) y el político (poder)6 ${ }^{6}$; son estos cuatro planos los que le brindan a la ideología su perfil propio como objeto de estudio.

Formalmente, la ideología tiene tres funciones clásicas, a saber: cognoscitiva (como medio de simplificación de la realidad para facilitar su comprensión), afectiva (como medio de gratificación psicológica mediante sentimientos de pertenencia e identidad) y normativa (como guía para el comportamiento político) ${ }^{7}$. Por lo cual no puede separarse la ideología del contexto de la acción colectiva que le da origen y la alimenta; forma con éste una relación dialéctica, en la que las construcciones ideológicas "están no solamente influenciadas por determinantes estructurales, materiales y simbólicos, sino que además contribuyen a su construcción" (Losada, 1998: 64).

Por otra parte, una concepción de la naturaleza del ser humano se encuentra implícita o explícitamente en los contenidos de la ideología, los cuales se expresan a través de símbolos, valores y creencias. Esos contenidos, desde luego, se revelan en el discurso a través del lenguaje ${ }^{8}$, que es modelado por la ideología (Volochinov, 1977: 15). Las creencias, que fundamentan una posición política, se objetivizan en el lenguaje. "El discurso político construye y significa una visión común del mundo" (Le Bart, 1998: 31).

En este sentido, "las palabras significan porque evocan o denotan de alguna manera una imagen" (Sartori, 1984: 35), y están presentes en todos los actos de comprensión e interpretación (Volochinov, 1977: 33). Estudiar los significados del lenguaje es estudiar el contenido del pensamiento de quien lo utiliza, es aproximarse a las representaciones sociales de los actores sociales.

El discurso político "se construye en efecto sobre el modo de un discurso moral, él opone fines legítimos y males que evitar, buenos y malos objetos" (Ansart, 1980: 66). En tal discurso, el hombre ideológico une hechos y valores, explicaciones y evaluaciones, hace coexistir lo moral y lo factual, en su descripción del mundo. Y sabemos que "el lenguaje no construye nunca sino pretendiendo describir" (Le Bart, 1998: 119).

Finalmente, la estructura y la lógica de la ideología se encuentra en la base de todo el proceso de conflicto simbólico por el poder de nominación de las relaciones que conforman la realidad social, de las estructuras cognitivas y las representaciones de los grupos sociales. La ideología brinda

5 Entendiendo por representaciones los esquemas cognitivos que guían la forma en la que el individuo experimenta el mundo, en que procesa la información.

$6 \quad$ La ideología y todos los procesos simbólicos que le acompañan y dan sentido a la acción desembocan en conflictos, lo que la coloca en el terreno de la lucha por el poder. Y "la noción de conflicto muestra cómo la necesidad, el deseo o el interés aunque no vengan dados por la consciencia que los experimenta, pueden tomar forma en la representación" (Foucault, 1992: 373).

7 Por lo que una ideología no es verdadera ni falsa; de lo que se trata es de comprender su grado de utilidad, si es eficiente 0 ineficiente, coherente 0 incoherente.

8 No olvidemos que por la existencia del lenguaje, el hombre puede "construirse todo un universo simbólico, al interior del cual puede igualmente construir alguna cosa como un saber (singularmente ese saber que él tiene de sí mismo)" (Foucault, 1992: 362). 
las claves para la comprensión de estos fenómenos, integra y articula los distintos planos y elementos que intervienen en ellos, da cuenta de los fenómenos relativos a la lucha por el poder simbólico ${ }^{9}$ y es clave en su comprensión.

\section{CONOCIMIENTO CIENTÍFICO Y USO DEL LENGUAJ E}

La ciencia es un conocimiento comprobable, obtenido conforme a procedimientos bien fundados y repetibles. En las ciencias sociales es el hombre, como ser social, el objeto de estudio, el cual "no se da al saber positivo más que en la medida en la que habla, trabaja y vive" (Foucault, 1992: 368). Pero en el estudio de su objeto, las ciencias sociales están condicionadas dado que el observador es de la misma naturaleza que lo observado y, en consecuencia, parte de la observación: el hecho humano no es, en general, directamente visible ${ }^{10}$ y la distancia objetiva al objeto no existe ${ }^{11}$.

Por otra parte, las ciencias sociales identifican la realidad con "un conjunto de relaciones invisibles, definidas las unas por relación a las otras" (Bourdieu, 1987: 150). En buena medida, estas relaciones son representaciones o producto de las representaciones, de las percepciones que los agentes sociales tienen del mundo social, las cuales se materializan a través de un proceso de nominación que contribuye a hacer las estructuras de ese mundo. En efecto, el mundo social podemos entenderlo como un ordenamiento por la palabra, un campo de significaciones (Pereña, 1999: 466). Y, en tal sentido, "la enunciación es un dominio básico de la acción social" (Abril, 1999: 448).

La ciencia, y la investigación empírica en particular, trabaja con datos, hechos, que provienen de la experiencia, que es la única que puede decidir la veracidad o falsedad de un enunciado científico (Popper, 1973: 39). Desde el punto de vista epistemológico, el hecho es eso que puede ser constatado, averiguado, que puede ser objeto de un entendimiento intersubjetivo al término de una verificación, una medida o un control experimental (Lecourt, 1999: 411). Ahora bien,

los hechos y los datos de las ciencias sociales no son tales, no son naturales, objetivos en el sentido más usual que se le suele asignar a estas nociones. No son sólo "sustantivos" o "positivos", ni están ahí "afuera en la realidad" a la espera de ser "captados" por nosotros los investigadores, sino que tales hechos y datos son construidos y producidos por los dispositivos tecnológicos y experimentales puestos en pie por los científicos-naturales y por los científicos-sociales (Conde, 1999: 63).

Para la producción y análisis de estos datos la investigación científica se vale de métodos explícitos, codificados y públicos que, por lo tanto, pueden evaluarse y repetirse. Todo el interés de la producción y procesamiento de estos datos -que como apunta Sartori, "no son otra cosa que informaciones colocadas dentro de palabras", trátese de investigaciones cuantitativas o cualitativas- no es otro

De acuerdo a Bourdieu, el poder simbólico es el poder de hacer cosas con palabras, es un poder de "consagración" o de "revelación". El poder simbólico, en tanto discurso performativo, está fundado sobre dos principios: se basa en la posesión de un capital simbólico y la eficacia de su utilización, que depende del grado en que la vision propuesta se funda en elementos de la realidad objetiva (Bourdieu, 1987: 164).

10 "Lo común que podemos descubrir en los hechos psicológicos, sociales y culturales, es que los unos y los otros no son cosas, sino un sentido que hay que aprehender, sentido que no aparece desde fuera" (Mucchielli, 1994: 16).

11 "El acto epistemológico mínimo consiste en generar una distinción (...) la información no se recoge sino que se genera como una nueva distinción, como resultado de una interacción que es, en sí misma, intervención. Intervenir es la condición de investigar" (Pakman, 1999: 361). 
que el de generar inferencias válidas sobre la vida social y política, sean inferencias descriptivas 0 causales, a partir de la información empírica que se tenga del mundo (King, Keohane y Verba: 18).

\section{LA INVESTIGACIÓN CUALITATIVA Y EL ESTUDIO DE LA IDEOLOGÍA}

Estos datos de los que hablamos, insumos indispensables del trabajo científico, son informaciones sobre el mundo recogidas de forma sistemática, y pueden ser tanto de tipo cualitativo como cuantitativo. En particular, los análisis cualitativos se orientan comúnmente hacia la búsqueda de formas y sentidos subyacentes ${ }^{12}$ a las conductas humanas y a los hechos sociales (Mucchielli, 1994: 15). En virtud de ello recordamos que "los procesos simbólicos y cognitivos de la mente humana quedan fuera de las respuestas conductuales registrables cuantitativamente" (Gutiérrez y Delgado, 1999: 142), por lo cual adoptamos una metodología cualitativa, por ser la más adecuada para el estudio del objeto que nos hemos propuesto analizar. Nuestra estrategia, además, apunta a una conexión entre teoría y datos con un fin básicamente descriptivo (cuando el objetivo es describir, sabemos que son dos las posibilidades: la primera, de carácter intensivo; la segunda, de carácter extensivo. La elección hecha nos sitúa frente a la primera, ante el estudio de caso) $)^{13}$.

Desde luego, conscientes estamos de los alcances y limitaciones de esta investigación. Sabemos que "toda elección metodológica construye su objeto de estudio. Selecciona la realidad que es pertinente y posible conocer" (Gutiérrez y Delgado, 1999: 142). Somos conscientes de que, siendo un estudio de caso, puede haber limitaciones para contrastar empíricamente la validez general de una hipótesis fuera del contexto del caso analizado ${ }^{14}$ y que no es posible -y tampoco es nuestra intención- a partir de esta investigación realizar inferencias que den lugar a generalizaciones teóricas ${ }^{15}$.

Ahora bien, si bien no corresponde hablar de fiabilidad en estudios de carácter cualitativo (dado que básicamente este término tiene una dimensión estadística), la noción de error es manejada a través del concepto de validez, sea interna o externa. Respecto a la primera, que vale tanto para los estudios cualitativos como cuantitativos, depende de la capacidad de que en la investigación ideas y hechos se correspondan ${ }^{16}$. Para lograr la segunda, hay varias alternativas, una de las cuales es la saturación ${ }^{17}$. "Cuando la saturación es alcanzada ella confiere una base sólida a la generalización; en una aproximación cualitativa ella cumple la misma función que la representatividad para la encuesta por cuestionario" (Mucchielli, 1994: 116).

"El sentido no es un dato sino una construcción social y, más precisamente, comunicativa o dialógica; no se trata pues de un objeto sino del proceso mismo en el que la relación intersubjetiva se objetiva y expresa" (Abril, 1999: 427).

13 "A case study may be used for exploratory, descriptive, or explanatory purposes (...) In the descriptive case, the purpose of a case study may be to find out and describe what happened in a single or select few situations. The emphasis is not on developing general explanations for what happened. According to Yin, case studies are most appropriately used to answer "how" or "why" questions" (Johnson y Joslyn, 1995: 144).

14 Es decir, lo que correspondería en los estudios cuantitativos a la noción de fiabilidad.

15 En particular, nos interesa el estudio de caso por su "capacidad de producir descripciones en profundidad, explicaciones detalladas de la lógica que guía el funcionamiento de procesos complejos, o nuevas hipótesis cuya validez y generalidad puedan ser puestas a prueba posteriormente analizando una muestra más amplia" (Anduiza, Crespo, Méndez, 1999: 63).

16 "Researchers routinely make complex choices about linking concepts to observations, that is, about connecting ideas with facts. Theses choices raise the basic question of measurement validity: Do the observations meaningfully capture the ideas contained in the concepts?" (Adcock y Collier, 2001: 529).

17 "La saturación es el fenómeno que aparece al cabo de un cierto tiempo en la investigación cualitativa cuando los datos que se recogen no son más nuevos. Todos los esfuerzos de colecta de información nueva resultan inútiles" (Mucchielli, 1994: 114). 
Finalmente, volviendo sobre la validez interna de toda investigación, lo importante es tener presente y claro que, sea cuantitativa o cualitativa, los conceptos han sido operacionalizados ${ }^{18}$ y los resultados deben ser interpretados en consideración a ellos:

valid measurement is achieved when scores (including the results of qualitative classification) meaningfully capture the ideas contained in the corresponding concept (...) our basic point is that measurement validation should focus on the relation between observations and the systematised concept; any potential disputes about the background concept should be set aside as an important but separate issue. With regard to scores, an obvious but crucial point must be stressed: scores are never examined in isolation; rather, they are interpreted and given meaning in relation to the systematised concept (Adcock y Collier, 2001: 530).

\section{EL ANÁLISIS DE CONTENIDO}

En primer término, el análisis de contenido es una técnica que permite el examen metódico, sistemático y objetivo del contenido de ciertos textos con vistas a clasificar e interpretar sus elementos constitutivos, los cuales no son totalmente accesibles a una lectura naive (Robert y Bouillaguel, 1997: 4). Hay aproximaciones múltiples y diversas en el análisis de contenido, pero todas convergen al menos en afirmar que "el discurso no es jamás esa fuente transparente que pretende ser" (Le Bart, 1998: 57). Las aproximaciones, en consecuencia, "varían en función de disciplinas sobre las que se apoyan, en función de tendencias al interior de esas disciplinas, en función también del tipo de fenómeno discursivo en el que se interesa" (Maingueneau, 1991: 16).

El análisis de contenido se ocupa, pues, de lo implícito. Y en esa búsqueda, se organiza a diferentes niveles de profundidad: desde lo más profundo (creencias, valores y normas), pasando por un sector intermedio (necesidades, emociones, predisposiciones y actitudes), hasta lo más externo (comportamientos y actos).

Desde el punto de vista empírico, hay dos posibilidades en el tratamiento de los datos: la lógica del tratamiento disyuntivo, la lógica de la clasificación,

que nos sirve para clasificar, un tratamiento lógico establecido por un criterio, que permite distribuir los datos en clases mutuamente excluyentes, que son a su vez exhaustivos (todos los datos deben ser clasificables);

y la lógica del tratamiento continuo, que es

una lógica (una sintaxis lógica) que puede denominarse de gradación. En este caso no queremos "cortar", sino más bien "arrimar": las diferencias son solamente de grados, de más y de menos. Con esta óptica los conceptos son transformados en variables, es decir que se hacen medibles de alguna manera (Sartori, 1984: 74).

En este estudio optamos por la primera alternativa, teniendo presente que las categorías que utilizamos son obviamente operacionalizaciones "que resultan del nivel de abstracción generalizante

18 Recordemos, "un concepto operacional es un concepto transferido y reducido a sus propiedades observables y definido por las operaciones que lo verifican" (Sartori, 1984: 66). 
al que el investigador decide situar su aprehensión del corpus para hacerlo pertinente en relación a sus objetivos" (Robert y Bouillaguel, 1997: 28).

En sentido general, los diversos análisis de contenido siguen 4 etapas básicas: 1) la búsqueda en el conjunto de casos posibles de un reagrupamiento en función de analogías de sistemas, 2) la búsqueda de elementos analógicos en las situaciones de cada agrupación de casos, 3) la sistematización del análisis a través de un cuadro-grille, 4) la formulación del sentido de una conducta, teniendo en cuenta el contexto situacional explícito (Mucchielli, 1994: 79).

Específicamente, para el caso que nos ocupa, nosotros nos hemos guiado por la metodología que proponen Robert y Bouillaguet ${ }^{19}$ para el análisis de contenido, contentiva de los siguientes pasos:

1. El preanálisis: constituye la etapa preliminar, de carácter intuitivo, que le brinda una primera dirección a la investigación.

2. La categorización: con el objeto de acceder a las significaciones no visibles inmediatamente, se diseña un cuadro-grille de categorías como instrumento para registrar elementos del corpus del discurso, para clasificar por categorías temáticas tales elementos y para realizar, posteriormente, comparaciones y porcentajes. Las categorías utilizadas deben, además, cumplir cuatro cualidades ${ }^{20}$.

3. Codificación y contaje de unidades: Como en todo proceso de recuento, hay que delimitar las unidades utilizadas para la disección del texto. En este sentido, hay que distinguir las

3.1. Unidades de registro, que designan el segmento determinado de contenido que se decide retener para hacerlo entrar en el cuadro-grille de análisis (palabras, frases, líneas, tema, etc.), y

3.2. Unidades de numeración, que designan la manera en que el analista va a contar cuando ha escogido le cuantificación ${ }^{21}$.

4. Interpretación de resultados: Esta etapa es la que permite evaluar la fecundidad de los dispositivos e hipótesis. Aquí se toma apoyo de los elementos revelados por la categorización para hacer una lectura del corpus, teniendo en cuenta que interpretar consiste en inferir, sacar consecuencias de los resultados obtenidos.

\section{IDEOLOGÍA, DISCURSO Y ANTIGLOBALIZACIÓN: EL CASO DE LA VÍA CAMPESINA}

Llegados a este punto y habiendo expuesto ya las bases de este trabajo, pasamos directamente a la descripción del protocolo de la investigación.

19 Ver Robert y Bouillaguet, pág. 25.

20 Son ellas: Pertinencia (capacidad de equilibrio entre el reflejo escrupuloso del corpus y la expresión de la problemática), Exhaustividad (todo el corpus debe encuentrarse registrado en el cuadro-grille), exclusividad (hay que evitar que mismos elementos de contenido pertenezcan a varias categorías), objetividad (como ya lo hemos señalado ampliamente, se trata de un término polémico y rebatible. Lo importante aquí es que el investigador aporte información adecuada de la construcción de las categorías y los indicadores) (Robert y Bouillaguet, 1997: 25).

${ }^{21}$ Aquí se debe hacer una acotación importante: la unidad de registro corresponde a eso que "cuenta", el número de ocurrencias, pero en el caso de la investigación cualitativa, en ocasiones, se trata más bien del reconocimiento de una ausencia o una presencia. 
Objeto del estudio: análisis del discurso político del movimiento social "La Vía Campesina" a partir de la teoría de la ideología.

Unidad de análisis: el discurso político.

Población diana: todos los documentos, declaraciones, informes y comunicados de la organización.

Población accesible: documentos colocados en la página web del movimiento.

Muestra: documento "Temas Eje".

Criterio de inclusión y exclusión para la selección de muestra: casos más apropiados para el objeto de estudio (Muestreo a Conveniencia ${ }^{22}$ ).

Categorización: como instrumento para el registro de los elementos pertinentes en el corpus (lo que hemos llamado planos o elementos estructurantes de la ideología) se han seleccionado tres categorías: representaciones, actitudes y acciones, las cuales han sido operacionalizadas de la siguiente manera:

1. Representaciones: cualquier enunciado que comporta una creencia que sirve de principio generador de toma de posición en los procesos simbólicos.

2. Actitudes: cualquier enunciado que evidencia una determinada predisposición u orientación de espíritu hacia un actor concerniente (positiva/aceptación, negativa/rechazo).

3. Acciones: cualquier enunciado que invoque o guíe una movilización en términos de lucha vinculada a relaciones de poder.

Codificación y resultados: en este proceso de recuento, la unidad utilizada para la disección del texto fue la frase ${ }^{23}$, en tanto unidad de registro ${ }^{24}$. Concretamente, en la categoría representaciones, nos guiamos por la "presuposición lógico-semántica" en tres de sus clases: los presupuestos existenciales (aquellos que, partiendo de una descripción, presuponen la existencia de la entidad que posee la cualidad descrita), presupuestos fácticos (aquellas preposiciones cuyo predicado expresan un hecho, que se presume cierto) y los presupuestos verbales (aquellos en los que el verbo describe la sucesión de dos estados, presumiéndose el anterior como producido) (Abril, 1999: 443). Hecho este procedimiento y tratado el contenido de la muestra, los resultados son los siguientes:

22 El "Muestreo a Conveniencia" es de carácter no probabilístico y los individuos o casos que se seleccionan son los considerados más apropiados para el objeto de estudio. Por otra parte, la revisión de otros documentos del movimiento no ha arrojado nuevos datos de interés, revelando así la presencia del fenómeno conocido como "saturación".

23 Ello lo hacemos en consideración de que "la unidad mínima de significado es la frase (unidad mínima de pensamiento, expresión semántica por excelencia). Hablar del sentido de una frase es hablar de la idea, de su idea, y la idea es siempre un lugar de encuentro... el cual descansa en la situación social, en el encuadre de una transparencia de sentido (la ideología)" (Pereña, 1999: 469).

24 Aun cuando esta etapa del análisis de contenido alude al modelo de utilización de códigos, aquí hemos utilizado un modelo inferencial en el tratamiento del corpus textual, en el entendido que el desfase entre las representaciones semánticas y los sentidos que se hacen efectivos en las prácticas comunicativas "no se salva con códigos, sino mediante inferencias o procesos de razonamiento de los interlocutores" (Abril, 1999: 442). 
TABLA 1: Agricultura Campesina Sostenible

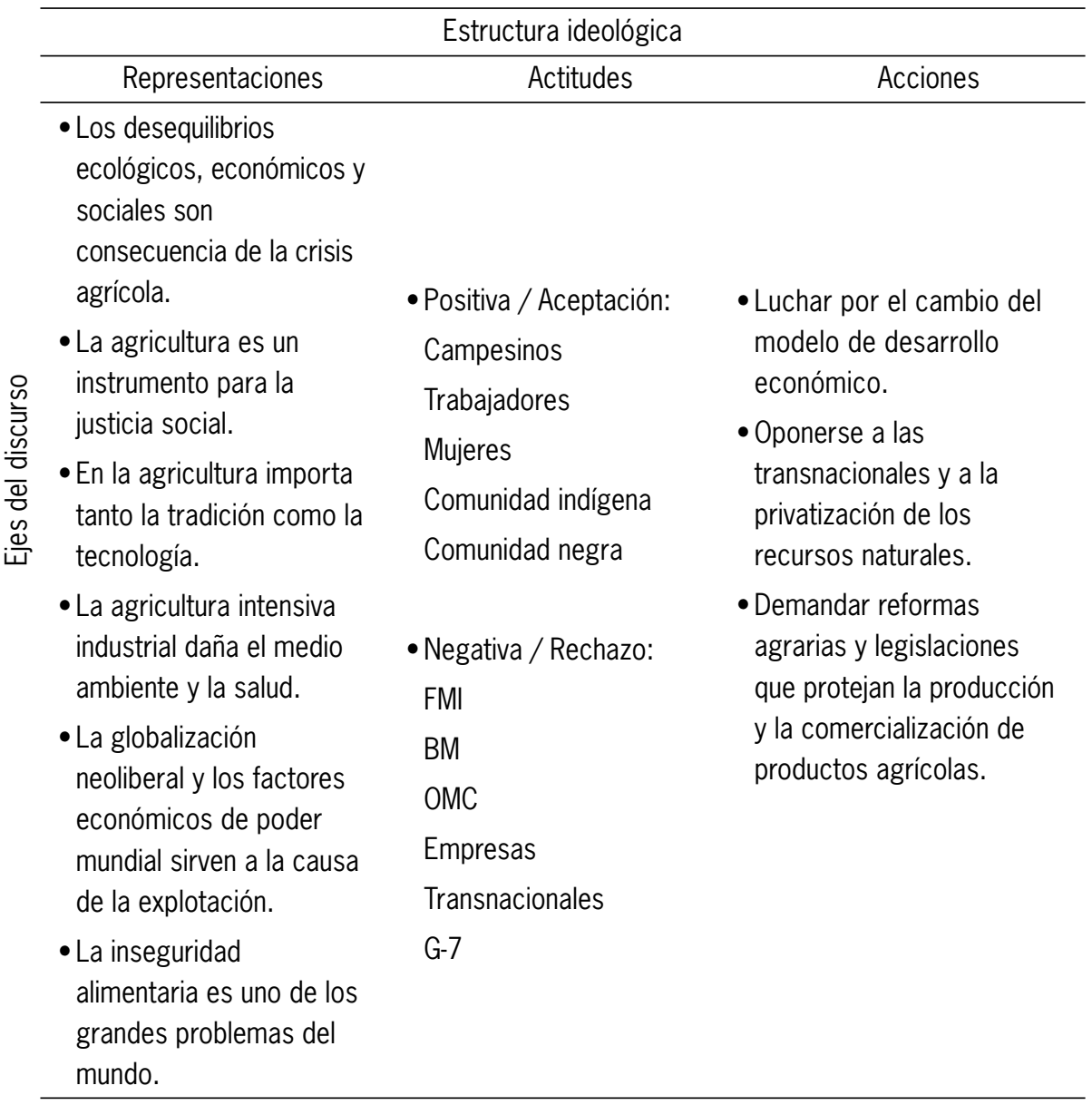


TABLA 2: Biodiversidad, Bioseguridad y Recursos Genéticos

\begin{tabular}{|c|c|c|}
\hline \multicolumn{3}{|c|}{ Estructura ideológica } \\
\hline Representaciones & Actitudes & Acciones \\
\hline $\begin{array}{l}\text { - La diversidad es la forma } \\
\text { de la vida y la base de la } \\
\text { riqueza. } \\
\text { - La biodiversidad es una } \\
\text { condición de la naturaleza } \\
\text { y de la sociedad. }\end{array}$ & & \\
\hline $\begin{array}{l}\text { - La riqueza biológica y } \\
\text { cultural del mundo se } \\
\text { concentra en los países } \\
\text { en desarrollo. } \\
\text { - Campesinos e indígenas } \\
\text { son "guardianes" de la } \\
\text { vida y la naturaleza. } \\
\text { - La agricultura persigue un } \\
\text { fin ético antes que } \\
\text { comercial. } \\
\text { - El libre mercado y el } \\
\text { capital internacional se } \\
\text { oponen al desarrollo } \\
\text { sustentable y la } \\
\text { seguridad alimentaria. } \\
\text { - El mejoramiento genético y } \\
\text { la evolución de las } \\
\text { especies en el campo son } \\
\text { consecuencia del trabajo } \\
\text { agrícola. }\end{array}$ & $\begin{array}{l}\text { - Positiva / Aceptación: } \\
\text { Comunidades campesina, } \\
\text { indígena, } \\
\text { negra, } \\
\text { Pescadores } \\
\text { Artesanos } \\
\text { - Negativa / Rechazo: } \\
\text { OMC } \\
\text { Capitalistas }\end{array}$ & $\begin{array}{l}\text { - Demandar la declaración } \\
\text { de moratoria a la } \\
\text { explotación, colección, } \\
\text { recolección, transporte y } \\
\text { modificación genética. } \\
\text { - Establecer mecanismos de } \\
\text { consulta y monitoreo para } \\
\text { proteger los derechos del } \\
\text { agricultor sobre los } \\
\text { recursos genéticos. } \\
\text { - Oponerse a la imposición } \\
\text { de modelos de vida o } \\
\text { desarrollo únicos. }\end{array}$ \\
\hline
\end{tabular}


TABLA 3: Recursos Genéticos y Derechos de Agricultores y Comunidades Rurales

\begin{tabular}{|c|c|c|}
\hline \multicolumn{3}{|c|}{ Estructura ideológica } \\
\hline Representaciones & Actitudes & Acciones \\
\hline $\begin{array}{l}\text { - La vida no es } \\
\text { comerciable. }\end{array}$ & & \\
\hline $\begin{array}{l}\text { - Los recursos genéticos } \\
\text { son patrimonio de la } \\
\text { humanidad. } \\
\text { - Los recursos naturales y } \\
\text { genéticos son los } \\
\text { elementos básicos de la } \\
\text { riqueza. }\end{array}$ & & $\begin{array}{l}\text { - Rechazar la propiedad } \\
\text { intelectual sobre toda } \\
\text { forma de vida y declarar } \\
\text { la moratoria de su } \\
\text { comercialización. }\end{array}$ \\
\hline $\begin{array}{l}\text { - La clonación humana } \\
\text { promueve la formación de } \\
\text { prototipos perfectos y } \\
\text { despierta el racismo y la } \\
\text { xenofobia. }\end{array}$ & & $\begin{array}{l}\text { - Proponer acuerdos sobre } \\
\text { bioseguridad y medio } \\
\text { ambiente. } \\
\text { - Organizar campañas } \\
\text { internacionales y }\end{array}$ \\
\hline $\begin{array}{l}\text { - Los campesinos han } \\
\text { protegido y mejorado la } \\
\text { diversidad genética a }\end{array}$ & $\begin{array}{l}\text { - Positiva / Aceptación: } \\
\text { Campesinos }\end{array}$ & $\begin{array}{l}\text { nacionales de información } \\
\text { sobre biodiversidad y } \\
\text { bioseguridad. }\end{array}$ \\
\hline $\begin{array}{l}\text { través de la historia. } \\
\text { - Los organismos } \\
\text { trangénicos rompen los } \\
\text { sistemas evolutivos y de } \\
\text { reproducción. }\end{array}$ & $\begin{array}{l}\text { Comunidades indígenas } \\
\text { - Negativa / Rechazo: } \\
\text { OMC }\end{array}$ & $\begin{array}{l}\text { - Organizar reuniones para } \\
\text { definir las estrategias e } \\
\text { intercambiar } \\
\text { experiencias. } \\
\text { - Constituir un mercado }\end{array}$ \\
\hline - Las patentes sobre & OMPI & alternativo para las \\
\hline materiales genéticos & OCDE & semillas y evitar que sean \\
\hline destruyen la soberanía y & G-7 & patentadas por las \\
\hline $\begin{array}{l}\text { - La manipulación genética } \\
\text { es una estrategia de los } \\
\text { países desarrollados para } \\
\text { sustituir materias primas } \\
\text { del tercer mundo. }\end{array}$ & $\begin{array}{l}\text { Empresas } \\
\text { Transnacionales }\end{array}$ & $\begin{array}{l}\text { - Organizar protesta contra } \\
\text { las semillas } \\
\text { genéticamente } \\
\text { manipuladas y las } \\
\text { transnacionales }\end{array}$ \\
\hline $\begin{array}{l}\text { - Los límites y controles } \\
\text { sobre recursos genéticos } \\
\text { ocasionará nuevas formas } \\
\text { de control sobre las } \\
\text { naciones y sus pueblos. }\end{array}$ & & agroquímicas. \\
\hline
\end{tabular}


TABLA 4: Derechos Humanos

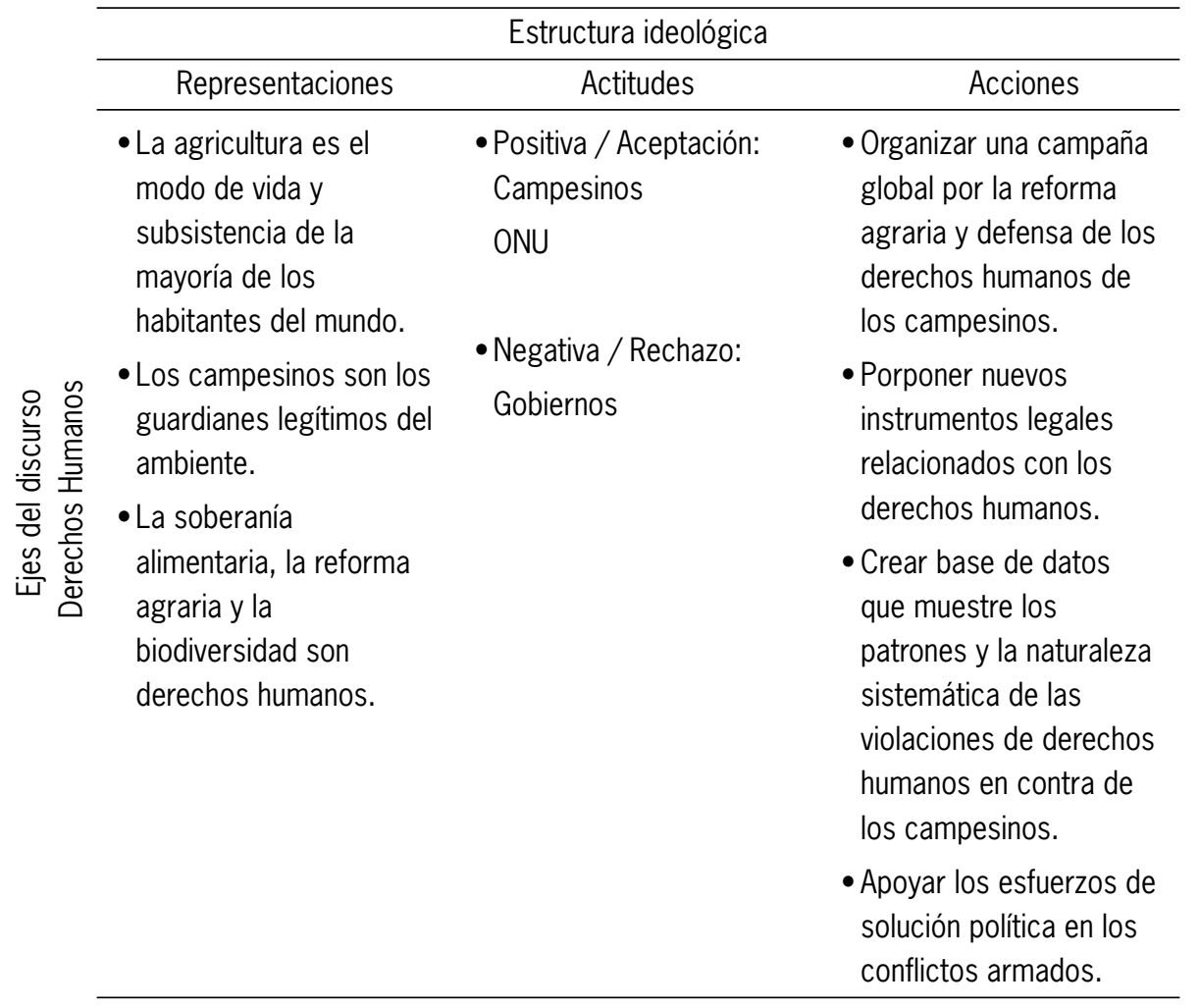


TABLA 5: Género

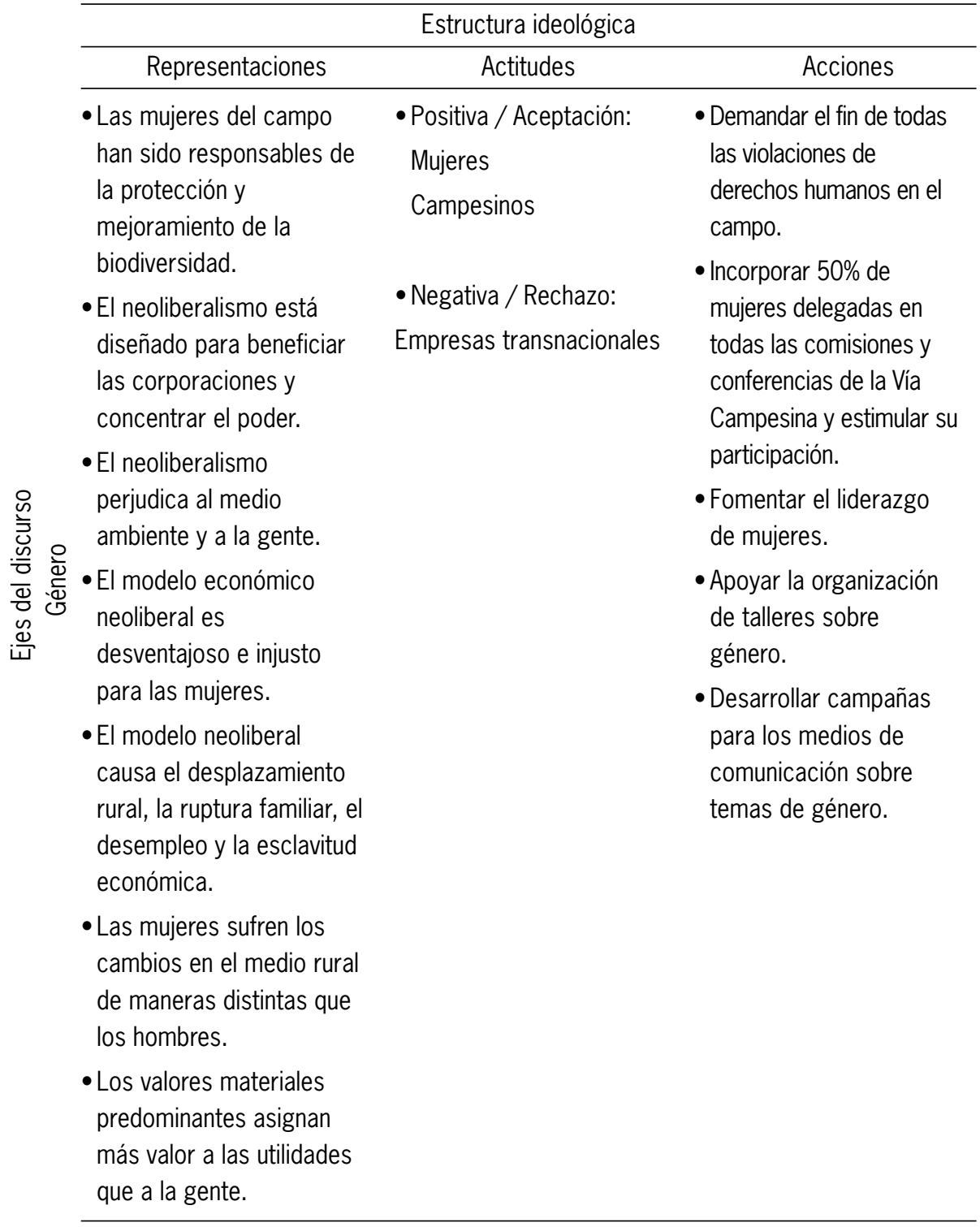


TABLA 6: Reforma Agraria y Cambios Sociales en el Campo

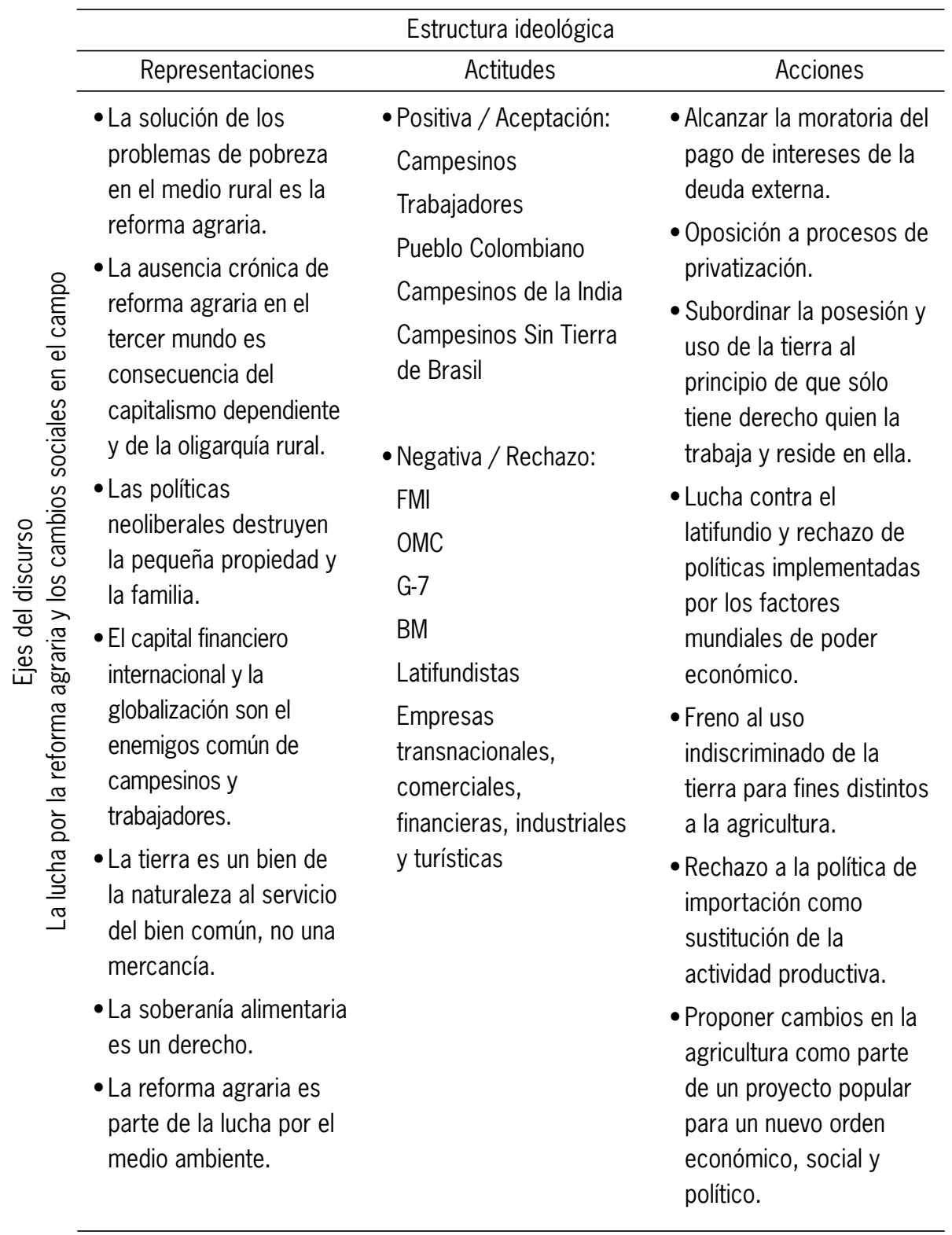


TABLA 7: Soberanía alimentaria y comercio internacional

\begin{tabular}{|c|c|c|c|}
\hline & & Estructura ideológica & \\
\hline & Representaciones & Actitudes & Acciones \\
\hline 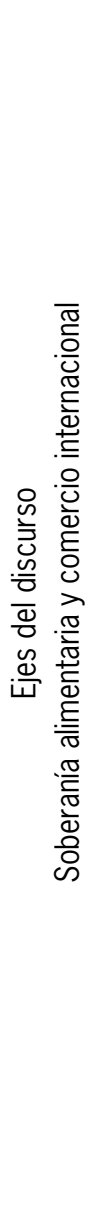 & $\begin{array}{l}\text { - La soberanía hace } \\
\text { posible la libertad. } \\
\text { - Para alcanzar la justicia } \\
\text { social es necesario ser } \\
\text { libre. } \\
\text { - La soberanía alimentaria } \\
\text { está amenazada por } \\
\text { intereses económicos } \\
\text { internacionales. } \\
\text { - Los precios mundiales } \\
\text { de los productos } \\
\text { agrícolas son artificiales } \\
\text { y resultan del dumping. } \\
\text { - Los agentes financieros } \\
\text { y económicos } \\
\text { internacionales no son } \\
\text { fiables. } \\
\text { - La soberanía alimentaria } \\
\text { garantiza una } \\
\text { producción sana, de } \\
\text { calidad y culturalmente } \\
\text { apropiada. }\end{array}$ & $\begin{array}{l}\text { - Positiva / Aceptación: } \\
\text { Campesinos } \\
\text { - Negativa / Rechazo: } \\
\text { FMI } \\
\text { OMC } \\
\text { BM }\end{array}$ & $\begin{array}{l}\text { - Desarrollar alianzas con } \\
\text { otros sectores sociales } \\
\text { para explicar la relación } \\
\text { entre la producción } \\
\text { campesina local y las } \\
\text { cuestiones } \\
\text { internacionales. } \\
\text { - Lanzar una campaña } \\
\text { mundial de acciones } \\
\text { contra el dumping. } \\
\text { - Estudiar los efectos de } \\
\text { los acuerdos de la OMC } \\
\text { sobre los trabajadores } \\
\text { agrícolas y los } \\
\text { inmigrantes. } \\
\text { - Proteger el mercado } \\
\text { interno contra } \\
\text { importaciones, impedir } \\
\text { los excedentes y eliminar } \\
\text { todas las ayudas a las } \\
\text { exportaciones. } \\
\text { - Debatir } \\
\text { internacionalmente el rol } \\
\text { de las multinacionales } \\
\text { en la destrucción de la } \\
\text { soberanía alimentaria. }\end{array}$ \\
\hline
\end{tabular}




\section{ANÁLISIS DE RESULTADOS}

Primeramente, los enunciados presentes en las tablas nos facilitan un mapa cognitivo en el que se encuentran presentes las creencias básicas que nutren los argumentos y toma de posición ante los temas que se han definido como "temas ejes" de la vía campesina. Estos, igualmente, nos permiten conocer la disposición que existe en tal discurso hacia aquellos actores que se nominan como los actores concernientes (actitudes) y las conductas que se adoptan en función de tales posiciones y disposiciones (acciones).

Con relación a la ideología (funciones), para comenzar, hay una comprensión simplificada de la realidad: "la solución de los problemas de pobreza en el medio rural es la reforma agraria" (ver Representaciones en Tabla 6, "Los desequilibrios ecológicos, económicos y sociales son consecuencia de la crisis agrícola" (ver Representaciones en Tabla 1. Asimismo, se evidencia una determinada afectividad hacia "aliados", como las comunidades indígenas (ver Actitudes en las tres primeras tablas, y "enemigos", como organismos financieros internacionales y empresas trasnacionales (prácticamente en todas las tablas). Además, los dos elementos anteriores convergen con los enunciados que sirven de guía para la acción, como el imperativo de "proteger el mercado interno contra importanciones, impedir los excedentes y eliminar todas las ayudas a las exportaciones" (ver Acciones en Tabla 7). Ello revela una lógica, que es la lógica propia de la ideología.

De igual modo, y no es menos importante, se encuentra presente todo el conjunto de elementos que conforman el hecho ideológico, elementos que describimos detalladamente en el marco teórico de esta investigación. Empecemos por la dinámica desarrollada en torno a la noción de grupo. En el discurso no sólo encontramos una autorrepresentación y una segmentación del grupo involucrado, que va desde una dimensión básica (campesinos), hasta una mayor (poblaciones rurales y/o conjunto de sectores desplazados) a partir de una cierta realidad (la globalización y el modelo neoliberal), sino también está presente esa dinámica propia de la ideología por la cual se expresan y defienden determinados intereses relativos al grupo, como el acceso a la tierra (la Tabla 6 "Reforma Agraria y Cambios Sociales en el Campo" es bastante representativo de lo que aquí se describe). Tampoco falta el esquema de polarización por el cual se definen los grupos antagónicos y sus intereses en oposición a los propios (esto puede observarse bastante bien en lo referente al tema de las patentes comerciales sobre materiales genéticos, en la Tabla 3).

Adicionalmente, respecto a esta noción de grupo, las representaciones presentes en el discurso aluden a cierta memoria fundadora ("Ios campesinos han protegido y mejorado la diversidad genética a través de la historia", ver Representaciones en Tabla 3), y aparecen las típicas palabras "marca" o "totem", que devienen en símbolos de los grupos ideológicos (reforma agraria, soberanía alimentaria, bioseguridad, etc.), las cuales recorren todo el discurso de la vía campesina, como puede apreciarse en las distintas tablas.

Por otra parte, en un modo característico de lo moral (justicia social, defensa de la vida, reivindicación de derechos), el discurso está construido sobre la base de la combinación de hechos y valores (el concepto de "inseguridad alimentaria" como problema mundial es un buen ejemplo, ver en Representaciones Tabla 1). Generalmente, las representaciones condenan o reivindican algo a lo que se le oponen acciones con fines legítimos que deben perseguirse ("el bien común", ver en Representaciones Tabla 6) o males que evitar (véase Representaciones y Acciones en Tabla 3). 
Tanto en el plano de las acciones como en el de las representaciones la noción de poder está siempre presente en términos de lucha (ver Acciones en las distintas tablas). Estas luchas 0 conflictos se despliegan básicamente sobre intereses y necesidades del grupo (la protección frente a la competencia, acceso a financiamiento y tecnología, propiedad de la tierra, etc.), que crean una "afinidad electiva" con las ideas que le sirven de base (soberanía alimentaria, derechos de los campesinos, reforma agraria) ${ }^{25}$.

Desde el punto de vista del sentido, hablar del contenido del texto como un todo es aludir a ese "algo con lo cual el texto funciona, en cierto modo, como instrumento". Por ello, este contenido, en cierta forma, está localizado fuera del texto, "en un plano distinto en relación con el cual ese texto define y revela su sentido"26 (Navarro y Díaz, 1999: 179). Así, en el caso del discurso de la vía campesina, el conjunto de enunciados contenidos en las categorías de las tablas presentadas adquieren sentido como expresión de un discurso ideológico (anti-globalización) que podríamos bien interpretarlo como partícipe del conflicto por el poder simbólico.

En el conjunto de enunciados contenidos en las tablas se "revelan" unas determinadas relaciones sociales, cuyas claves hay que pensarlas en función de los intereses y necesidades del grupo al cual tal discurso representa (campesinos/desplazados) y en oposición a las aspiraciones e intereses de los grupos considerados adversarios (empresas, financistas, organizaciones internacionales). Obviamente, esta disputa por el poder simbólico de imponer una nominación de la realidad (pensemos en los conceptos de "diversidad genética", "biodiversidad", "seguridad alimentaria") no es sólo hacerlo con autoridad (la de quienes luchan por la justicia, en contra de poderosos intereses económicos, en el nombre de grandes mayorías, como herederos de legados culturales), es sobre todo hacerlo con eficacia en relación a una realidad objetiva: el crecimiento de la pobreza y la desigualdad (en el campo, en este caso).

\section{CONCLUSIONES}

Son muchas las implicaciones y relaciones que pueden intentar establecerse a partir de los resultados obtenidos, y es natural que así sea dada la naturaleza y objetivo general de este estudio. No obstante, al inicio del mismo señalamos los aspectos fundamentales sobre los que pretendíamos centrarnos y son estos aspectos sobre los que fijaremos las conclusiones del trabajo, dejando a estudios posteriores el desarrollo de algunas vetas que consideramos de interés.

En este sentido, pensamos que esta investigación pone en evidencia los elementos estructurantes del discurso político de la vía campesina en su vertiente ideológica, así como la lógica que estos siguen. Hemos dado cuenta, además, de las representaciones principales que le sirven de base a este discurso y apuntado relaciones que articulan sentidos desde los cuales puede interpretarse el mismo en función de la realidad social de la que forma parte y los conflictos que en ella se libran, materiales y simbólicos.

25 Sobre este punto vale la pena un comentario adicional. Es cuando menos curiosa la modesta presencia de explícitas menciones al poder público (en la categoría Actitudes apenas se registra negativamente la presencia de "Gobiernos" en la Tabla 4 "Derechos Humanos", lo cual parece indicar que la concepción que se tiene del poder es fundamentalmente atada a lo económico y supranacional). No obstante, en las estrategias que se desprenden del programa de acciones es evidente que se considera a los mecanismos legales (legislación) una forma efectiva de lucha.

26 El sentido tiene una dimensión semántica (significado, carácter simbólico) y, por la otra, es una geometría del deseo (orientación, relación a un fin) (García Selgas, 1999: 495). 
Por otra parte, nos parece provechoso -en consideración a los resultados obtenidos- el estudio de los procesos de cambio a partir del discurso, especialmente las luchas por la nominación de los factores que en ellos intervienen. Esta investigación avala, asimismo, la tesis que sostiene que los movimientos sociales constituyen espacios desde los cuales observar la dinámica social (en este caso, los movimientos antiglobalización y la resistencia a los modelos económicos basados en la competencia y el libre mercado).

En virtud de los elementos básicos de la ideología presentes en este discurso (particularmente en lo referente a la construcción de identidad y cohesión de los grupos y a cómo ello se conecta con los conflictos de intereses y las dinámicas sociales), el trabajo ofrece insumos para la especulación y la formulación de hipótesis, sea con referencia al modelo teórico de análisis de la ideología 0 al entendimiento y comprensión del horizonte de representaciones de otros movimientos sociales. En un sentido u otro, será necesario sin duda explorar las posibilidades de hacer generalizaciones a partir de trabajos comparativos.

Esta investigación deja, a nuestro criterio, preguntas e interrogantes que surgen en estudios iniciales como éste, cuyos modestos fines son principalmente descriptivos y han sido ya suficientemente expuestos. Consideramos, no obstante, que tales interrogantes y posibles vacíos no sólo son los costos de una primera aproximación, sino también insumos que, en sí mismos, estimulan la búsqueda de nuevos caminos y soluciones al conocimiento de este dominio.

\section{REFERENCIAS}

Abril, Gonzalo. 1999. "Análisis semiótico del discurso". En Métodos y técnicas cualitativas de investigación en ciencias sociales, editado por Juan Delgado y Juan Gutiérrez. Madrid: Síntesis.

Adcock, Robert y David Collier. 2001. "Measurement validity: a shared standard for qualitative and quantitative research". American Political Science Review, 95 (3): 529-546.

Anduiza, Eva, Mónica Méndez e Ismael Crespo. 1999. Metodología de la ciencia política. Madrid: CIS.

Ansart, Pierre. 1980. "Les sujets dans l'idéologie". En Analyse de l'idéologie, editado por G. Duprat. Paris: Editions Galilée. Bobbio, Norberto, Niccola Matteucci y Gianfranco Pasquino. 1998. Diccionario de política. México: Siglo XXI Editores.

Bourdieu, Pierre. 1982. Ce que parler veut dire. Paris: Fayard.

Bourdieu, Pierre. 1987. Choses dites. Paris: Les éditions de minuit.

Conde, Fernando. 1999. "Las perspectivas metodológicas cualitativa y cuantitativa en el contexto de la historia de las ciencias". En Métodos y técnicas cualitativas de investigación en ciencias sociales, editado por Juan Delgado y Juan Gutiérrez. Madrid: Síntesis.

Dávila, Andrés. 1999. "Las perspectivas metodológicas cualitativa y cuantitativa en las ciencias sociales: debate teórico e implicaciones praxeológicas". En Métodos y técnicas cualitativas de investigación en ciencias sociales, editado por Juan Delgado y Juan Gutiérrez. Madrid: Síntesis.

García Selgas, Fernando. 1999. "Análisis del sentido de la acción: el trasfondo de la intencionalidad". En Métodos y técnicas cualitativas de investigación en ciencias sociales, editado por Juan Delgado y Juan Gutiérrez. Madrid: Síntesis.

Gutiérrez Juan y Juan Delgado. 1999. "Teoría de la observación”. En Métodos y técnicas cualitativas de investigación en ciencias sociales, editado por Juan Delgado y Juan Gutiérrez. Madrid: Síntesis.

Foucault, Michel. 1992. Les mots et les choses: une archéologie des science humaines. Paris: Gallimard. Johnson, James y Richard Joslyn. 1995. Political science research methods. Washington: Congressional Quarterly Inc. Ibarra, Pedro y Benjamín Tejerina (eds). 1998. Los movimientos sociales. Transformaciones políticas y cambio cultural. Madrid: Trotta. 
King, Gary, Robert Keohane y Sidney Verba. 1994. El diseño de la investigación social. Madrid: Alianza.

Le Bart, Christian. 1998. Le discours politique. Paris: Presses Universitaires de France.

Lecourt, Dominique. 1999. Dictionnaire d'histoire et philosophie des sciences. Paris: Presses Universitaires de France.

Lozada, Marta. 1998. "Ideología y militancia: ¿fin del compromiso?". Revista Venezolana de Ciencia Política 14 (julio-diciembre). Maingueneau, Dominique. 1991. L'analyse du discours: introduction aux lectures de l'archive. Paris: Hachette.

Mardones, José María (coord.). 1996. Diez palabras clave sobre movimientos sociales. Estella: Verbo Divino.

Mees, Ludger. 1998. “¿Vino viejo en odres nuevos? Continuidades y discontinuidades en la historia de los movimientos sociales”.

En Los movimientos sociales. Transformaciones políticas y cambio cultural, editado por Pedro Ibarra y Benjamín Tejerina. Madrid: Trotta.

Mucchielli, Alex. 1994. Les méthodes qualitatives. Paris: Presses Universitaires de France.

Navarro, Pablo y Capitolina Díaz. 1999. "Análisis de contenido". En Métodos y técnicas cualitativas de investigación en ciencias sociales, editado por Juan Delgado y Juan Gutiérrez. Madrid: Síntesis.

Ortí, Alfonso. 1999. "La confrontación de modelos y niveles epistemológicos en la génesis e historia de la investigación social". En Métodos y técnicas cualitativas de investigación en ciencias sociales, editado por Juan Delgado y Juan Gutiérrez. Madrid: Síntesis.

Pakman, Marcelo. 1999. "Investigación e intervención en grupos familiares. Una perspectiva contructivista". En Métodos y técnicas cualitativas de investigación en ciencias sociales, editado por Juan Delgado y Juan Gutiérrez. Madrid: Síntesis.

Pereña, Francisco. 1999. "Formación discursiva, semántica y psicoanálisis". En Métodos y técnicas cualitativas de investigación en ciencias sociales, editado por Juan Delgado y Juan Gutiérrez. Madrid: Síntesis.

Popper, Karl. 1973. La logique de la découverte scientifique. Paris: Payot.

Raschke, Joachim. 1994. "Sobre el concepto de movimiento social". Zona Abierta 69. Madrid.

Ricoeur, Paul. 1997. L'idéologie et la utopie. Paris: Editions du seuil.

Robert, André y Annick Bouilliaguet. 1997. L'analyse de contenu. Paris: Presses Universitaires de France.

Sartori, Giovanni. 1984. La política. Lógica y método de las ciencias sociales. México: FCE.

Tarrow, Sydney. 1999. "Estado y oportunidades: la estructuración política de los movimientos sociales". En Movimientos sociales: perspectivas comparadas, editado por Doug McAdam, John McCarthy y Mayer Zald. Madrid: Istmo.

Van Dijk, Teun. 1999. Ideología. Barcelona: Gedisa.

Volochinov, Valentin. 1977. Le marxisme et la philosophie du langage. Paris: Les éditions de minuit.

J orge Lazo Cividanes es candidato a doctor en ciencia política en la Universidad de Salamanca, España. Previamente, obtuvo los títulos de licenciado en comunicación social (Universidad Central de Venezuela) y magíster en ciencia política (Universidad Simón Bolívar). Además de la actividad docente, se ha desempeñado como consultor en el área de comunicaciones estratégicas, y ha publicado varios trabajos, tanto teóricos como empíricos, relativos al estudio de la ideología, el análisis del discurso y el pensamiento políticos en América Latina.

(E-mail: jorge@yahoo.com). 